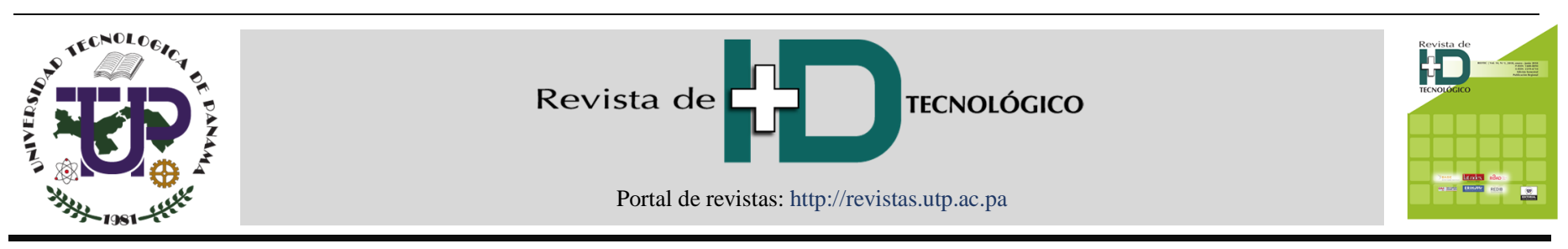

\title{
Diseño preliminar parte I, de un túnel de viento de circuito abierto (tipo Eiffel) para números de Reynolds de $3.3 \times 10^{6} \mathrm{~m}^{-1}$
}

\section{Preliminary design part I, of an open circuit wind tunnel (type Eiffel) for Reynolds of $3.3 \times 10^{6} \mathrm{~m}^{-1}$}

\author{
Omar Cornejo Luna ${ }^{1 *}$, Luis Mogollón ${ }^{1}$, Humberto Álvarez ${ }^{1}$ \\ ${ }^{1}$ Centro de Investigación e Innovación Eléctrica, Mecánica y de la Industria (CINEMI), Universidad Tecnológica de Panamá, Panamá \\ *Autor de correspondencia: omar.cornejo@utp.ac.pa
}

RESUMEN-El propósito de este trabajo, es el prediseño de un túnel de viento de circuito abierto subsónico para la Universidad Tecnológica de Panamá; para el desarrollo de futuras investigaciones como lo son el diseño de pequeños aerogeneradores e investigaciones de dispersión de emisiones contaminantes, entre otros. Para ello se requirió realizar una extensa investigación de los modelos existentes y las limitaciones de los diversos componentes que conforman un túnel de viento con esta configuración, basándose en las pautas y lineamiento existentes a la fecha y con el apoyo de la dinámica de fluidos computacional. El empleo de los modelos y el apoyo de dichos métodos numéricos permitió obtener un prediseño funcional que teóricamente satisface los requerimientos iniciales, y permitió desarrollar una correlación para el diseño de la contracción, propiciando nuevos caminos de investigación con el fin de mejorar el rendimiento del ventilador axial.

Palabras clave-Dinámica de fluidos computacional, contracción, difusor, malla, sección de pruebas, túnel de viento, ventilador.

ABSTRACT - The purpose of this work is pre-design a subsonic open-circuit wind tunnel for the Technological University of Panama; for the development of future researchs such as the design of small wind turbines and research on the dispersion of polluting emissions, among others. For them, it was necessary to carry out an extensive investigation of the existing models and their limitations of the various components that make up a wind tunnel with this configuration, based on the existing guidelines to date and with the support of computational fluid dynamics. The use of the models and the support of these numerical methods allowed us to obtain a theoretically functional design that satisfies our initial requirements, propitious to develop a correlation for the design of the contraction, fostering new research paths in order to improve the performance of the fan axial.

Keywords - Computational fluid dynamic, contraction, diffusor, mesh, test section, wind tunnel, fan.

\section{Introducción}

La optimización y desarrollo de aerogeneradores, requiere de herramientas de investigación que permitan obtener flujos de aire que simulen las condiciones reales a las cuales están expuesto, a través del empleo de un modelo a escala.

El túnel de viento es una herramienta de investigación en mecánica de fluidos, específicamente de gran utilidad en aerodinámica experimental que ha permitido resolver un amplio rango de problemas, fenómenos que involucra la interacción con el aire en movimiento.

Su empleo inicial permitió a los hermanos Wright conocer información básica sobre arrastre producida por el ala, la sustentación y el centro de presión necesarias para el correcto diseño de su aeronave. Otros investigadores como lo son Reynolds, Tsiolkovsky, Lilienthal, Langley, Prantdl, Von Karman e Eiffel, entre otros, llevaron a cabo investigación con esta herramienta.

Entre las décadas del 30 y el 40 del pasado siglo, se produjo un rápido avance en el diseño y construcción. Esta herramienta posee cerca de 147 años desde que fue construida por primera vez en 1871 por parte del Wenham [1], existiendo una gran cantidad de investigaciones referente a su diseño y materiales empleados [2], actualmente aún en constante evolución.

Existen varios tipos de túneles de viento [3-12], lo cual depende del tipo de experimentos que se pretende llevar adelante, siendo los más populares los túneles de vientos de circuito abierto, llamados también túnel de viento de Eiffel y los túneles de viento de circuito cerrado. Basados en nuestros requerimientos y necesidades, se seleccionó un túnel de viento de circuito abierto o túnel de viento tipo Eiffel subsónico con un número de Mach < 0.4 [4].

Citación: O. Cornejo, L. Mogollón y H. Alvarez, "Diseño preliminar parte I, de un túnel de viento de circuito abierto (tipo Eiffel) para números de Reynolds de 3.3 X106 m-1", Revista de I+D Tecnológico, vol. 15, no. 1, pp. (5-16), 2019.

Tipo de artículo: Original. Recibido: 23 de mayo de 2018. Recibido con correcciones: 19 de noviembre de 2018. Aceptado: 19 de noviembre de 2018. DOI https://doi.org/10.33412/idt.v15.1.2091

Copyright: This is an open access article under the CC BY-NC-SA 4.0 license (https://creativecommons.org/licenses/by-nc-sa/4.0/). 
Actualmente solo se tienen directrices, más o menos establecidas, para el diseño y construcción de túneles de viento de diversos tipos. La mayoría de la bibliografía consultada está basada en experimentación, que con el tiempo han llegado a ser, algunas de ellas, directrices[4] [13]. A través de métodos numéricos computacionales, es posible llevar a cabo un prediseño de un túnel de viento abierto tipo Eiffel.

En este trabajo se emplearán métodos numéricos computacionales. Específicamente, Dinámica de fluido Computacional (CFD) en conjunto con herramientas CAD, con el fin de optimizar la geometría de las diversas partes que conforman el túnel de viento de tipo Eiffel para velocidades comprendidas entre los $4 \mathrm{~m} / \mathrm{s}$ y los 65 $\mathrm{m} / \mathrm{s}$. En este prediseño de tipo preliminar no se tiene en cuenta las paredes del recinto donde estar instalado, la cámara de tranquilización y la toma de aire del mismo.

\section{Fundamentos teóricos y características de diseño}

Los túneles de viento abiertos poseen cinco (5)

componentes principales:

1. La zona de acondicionamiento del flujo

2. La contracción

3. La sección de pruebas

4. El difusor

5. El ventilador.

La zona de acondicionamiento del flujo se encuentra agua arriba de la sección de prueba y contiene "malla de abeja" para reducir la turbulencia, con la finalidad de producir una corriente de aire lo más uniforme posible. La contracción permite tomar un volumen grande de aire a baja velocidad reduciendo a un volumen pequeño de aire de alta velocidad sin crear turbulencia. Por otro lado, la sección de pruebas es la zona donde se instala el modelo y su correspondiente instrumentación.

El difusor tiene como objetivo transformar la energía cinética en energía de presión de manera eficiente, mientras que el ventilador suministra la energía necesaria para la corriente de aire y suple la pérdida producida por la corriente de aire a través de las secciones anteriores.

\subsection{Requerimiento del túnel de viento y configuración del túnel de viento}

Los requisitos condicionan la configuración y las dimensiones; por otro lado, los recursos económicos y materiales existentes y métodos de manufactura poseen un gran peso durante el proceso de selección basado en casa de la calidad.

Entre los requisitos empleados durante nuestra selección del tipo de túnel a diseñar, se tiene:

- Poseer un número de Reynolds superior a $1.0 \mathrm{E}+6 \mathrm{~m}^{-1}$.

- Poder crear velocidades comprendidas entre 4 $\mathrm{m} / \mathrm{s}$ hasta $65 \mathrm{~m} / \mathrm{s}$.

- Costos bajos de construcción.

- Posibilidad de hacer visualización con humos.

- Facilidad de manufactura.

Para nuestro caso en particular, el túnel de circuito abierto resultó ser la mejor opción. Cumpliendo con la mayoría de los requerimientos antes expuestos.

Con el objetivo de poder emplear objetos cuya envergadura máxima fuera $0.8 \mathrm{~m}$, se diseñó la sección de prueba con dimensiones de $0.92 \mathrm{~m} \times 0.92 \mathrm{~m}$ y una longitud de $2 \mathrm{~m}$; esto permite obtener números de Reynolds mayores a $3.0 \times 10^{6} 1 / \mathrm{m}$ para maquetas de $0.8 \mathrm{~m}$ de envergadura.

Tabla 1. Propiedades del aire a diferentes temperaturas

\begin{tabular}{cccc}
\hline \hline Temperatura & $\mathbf{1 5}^{\circ} \mathbf{C}$ & $\mathbf{2 0}^{\circ} \mathbf{C}$ & $\mathbf{3 0}^{\circ} \mathbf{C}$ \\
\hline \hline $\boldsymbol{\mu}(\mathrm{Kg} / \mathrm{m}-\mathrm{s})$ & $1.80 \mathrm{E}-5$ & $1.83 \mathrm{E}-5$ & $1.83 \mathrm{E}-5$ \\
$\boldsymbol{\rho}\left(\mathrm{Kg} / \mathrm{m}^{3}\right)$ & 1.225 & 1.204 & 1.164 \\
$\mathbf{v}(\mathrm{m} / \mathrm{s})$ & 65 & 65 & 65 \\
$\mathbf{D}(\mathrm{m})$ & 0.8 & 0.8 & 0.8 \\
$\boldsymbol{R e}(1 / \mathrm{m})$ & $3.53 \mathrm{E}+6$ & $3.43 \mathrm{E}+6$ & $3.31 \mathrm{E}+6$ \\
\hline \hline
\end{tabular}

\subsection{Configuración estructural del túnel de viento}

Para determinar las diversas zonas que componen el túnel de viento se analizaron diversos modelos propuestos por otros investigadores.

Se inició dimensionando la sección de prueba para poseer un área antes mencionada, para velocidad máxima expuesta con anterioridad. Ver tabla 1.

El diseño de la contracción es muy sensitiva y demorada; requirió más de tres meses de estudios de diversos modelos, propuestos por diversos 
investigadores [15-23]. Se procedió a desarrollar la geometría propuesta y llevar a cabo la simulación de la misma. Las limitaciones de algunos de estos modelos reducían significativamente las cargas de presión, la pérdida de uniformidad del flujo, se producían desprendimiento en la contracción y zona de prueba, siendo el modelo más prometedor el presentado por [14].

A partir de este modelo se procedió a realizar diversas modificaciones geométricas hasta obtener el siguiente modelo propio, el cual es el empleado en nuestro actual estudio.

$y(x)=0.8688+1.0197 \cdot x-1.0992 \cdot x^{2}+0.252 \cdot x^{3}$

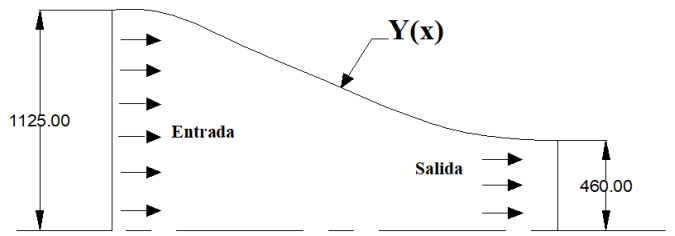

Figura 1. Dimensiones y modelo de la contracción.

La metodología empleada en el desarrollo de la Contracción del túnel de viento, siguiente; nos permitió evaluar la gran cantidad de correlación para el diseño de la contracción.

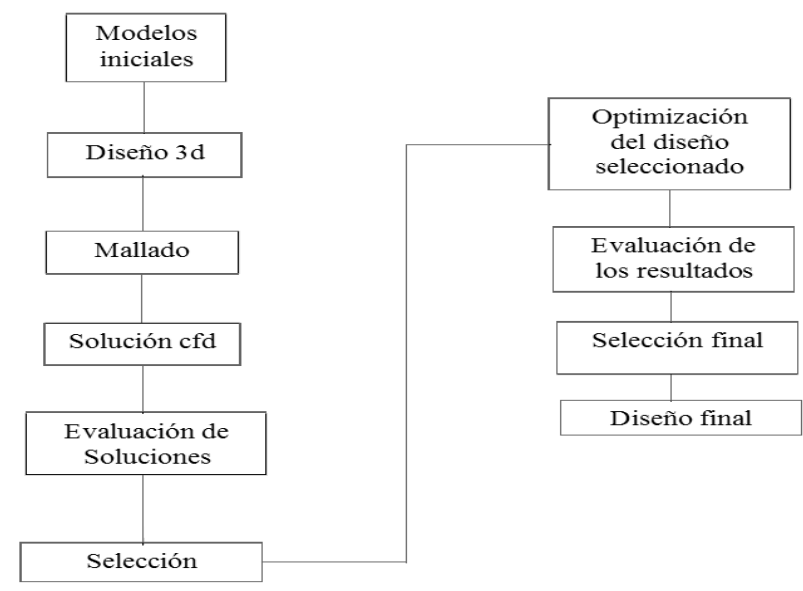

Figura 2. Metodología de desarrollo de la contracción.

Posteriormente se procedió a determinar las dimensiones del difusor, para ellos se empleó el modelo propuesto por [4], teniendo en cuenta el radio hidráulico de la sección de prueba, el ángulo máximo sugerido $6^{\circ}$ para todo el difusor, la relación $\left(A_{R}\right)$ entre el área de la salida del difusor y el área de la sección de prueba $\left(D_{1}\right)$.

$$
\theta_{e}=\operatorname{actan}\left(\frac{1}{2} \frac{\sqrt{A_{R}}-1}{\frac{L}{D_{1}}}\right)
$$

Para el área de salida del difusor se empleó una relación de área 2.2, las sugerencias más modernas como [2], sitúan esta relación de área entre $1.5<A_{R}<6$. Esto permitió obtener una longitud del difusor (L) de $4.3 \mathrm{~m}$ de largo, logrando el correcto desarrollo del perfil de velocidad del fluido.

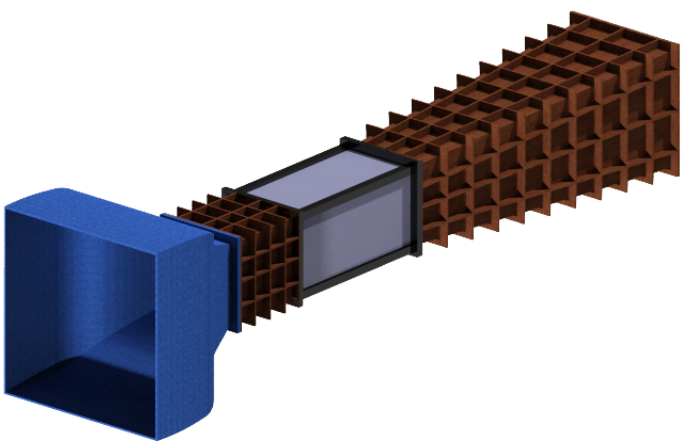

Figura 3. Túnel de viento.

La selección del ventilador obedece a las necesidades de flujo y las pérdidas que este debe superar, producida por las diversas secciones que comprenden el túnel de viento. Para ello se procedió a determinar el flujo de aire en la sección de prueba para condiciones de temperatura de $30^{\circ} \mathrm{C}$, siendo este flujo $51.55 \mathrm{~m}^{3} / \mathrm{s}$ y la pérdida a través de los componentes del túnel de viento, como se observará en las siguientes secciones.

A partir de esto se seleccionó un ventilador axial, el cual posee un motor eléctrico de $300 \mathrm{Hp}$ y capacidad de 0.55 psi (0.038 bar). Este tipo de ventiladores poseen aplicaciones donde se requiere mover grandes cantidades de aire como baja resistencia al flujo y producir un incremento de presión máximo de 1.05 bar.

La eficiencia del ventilador se puede obtener empleando la siguiente ecuación:

$$
\eta=1-\left[\frac{\epsilon_{\mathrm{m}}}{\varphi} \cdot \frac{2}{3} \cdot \frac{1-\vartheta^{3}}{1-\vartheta^{2}}+\left(1-\eta_{\mathrm{p}}\right) \cdot \frac{\varphi^{2}}{\psi}\right]
$$

Donde $\epsilon_{\mathrm{m}}$ se refiere al índice de frotamiento, $\varphi$ у $\psi$ son coeficientes de flujo y el coeficiente de trabajo, $v$ es el coeficiente de rozamiento, $\eta_{p}$ eficiencia isentrópica [24][25]. 


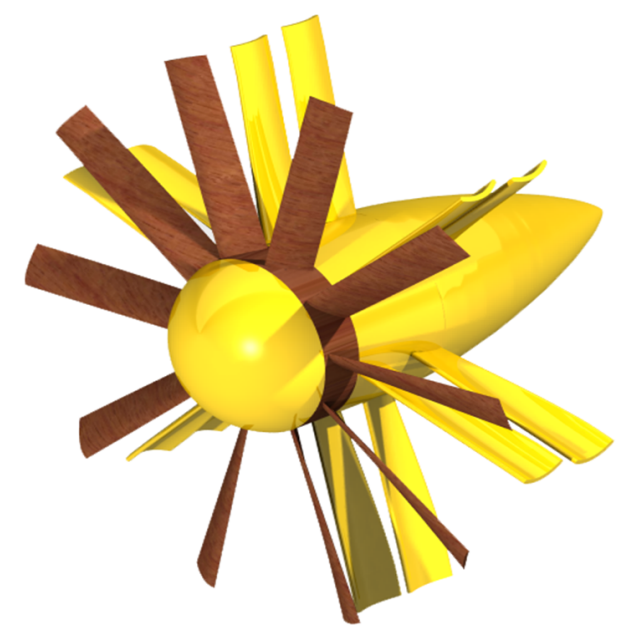

Figura 4. Ventilador axial.

En la tabla siguiente se muestra las características técnicas del ventilador seleccionado.

Tabla 2. Características del Ventilador Axial

\begin{tabular}{|c|c|}
\hline \multicolumn{2}{|c|}{ Características del ventilador } \\
\hline$\psi$ & 0.514 \\
\hline$\varphi$ & 0.329 \\
\hline ๆvol. (\%) & 93.8 \\
\hline 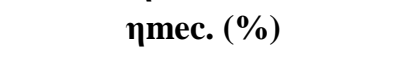 & 91 \\
\hline paero. (\%) & 80 \\
\hline$\Delta \mathbf{P}($ bar $)$ & 0.038 \\
\hline $\mathbf{Q}(\mathbf{m} 3 / \mathbf{h})$ & $\begin{array}{l}185,5 \\
00\end{array}$ \\
\hline \# Alabes & 10 \\
\hline Rpm max del motor & 1750 \\
\hline Perfil Aerodinámico Naca & 6309 \\
\hline
\end{tabular}

Nuestro ventilador axial, posee un cuerpo aerodinámico, el motor eléctrico dentro de una carcasa fuselada y Vanes Guide (straighteners) o enderezador de flujo, que permite disminuir torbellino (swirl) producido por las alabes del rotor del ventilador. El diseño y desarrollo de ventiladores axiales no es algo trivial [2840].

Para este ventilador se tiene un rendimiento volumétrico como lo muestra la figura 5 .

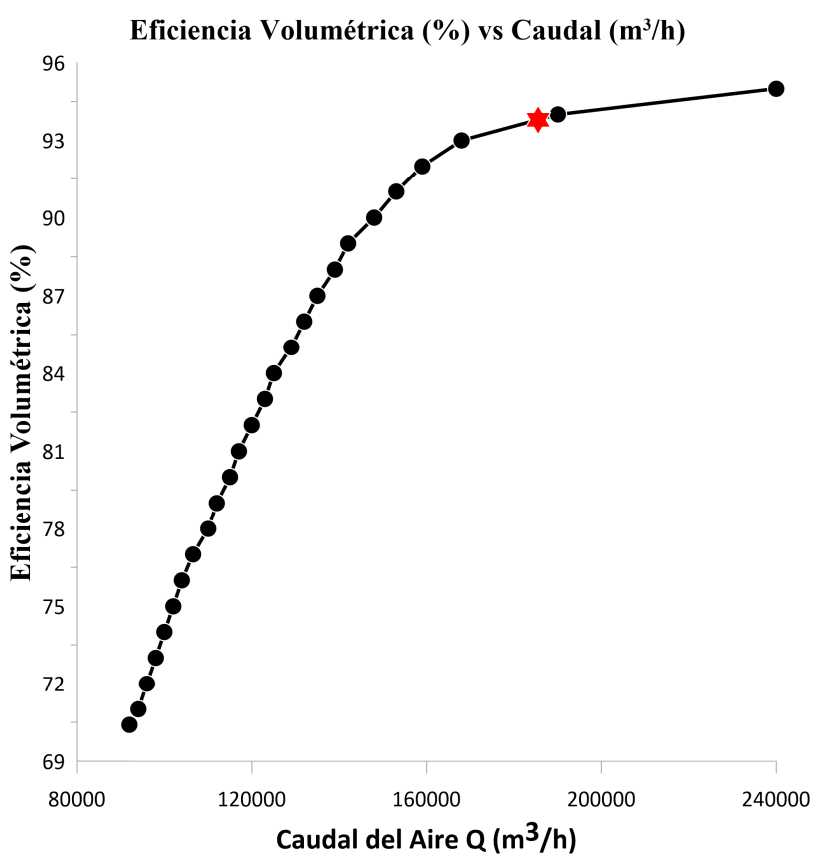

Figura 5. Eficiencia volumétrica del ventilador axial.

\subsection{Cálculo de pérdidas en componentes del túnel de viento}

Para la correcta selección o diseño del ventilador, el cual creará la corriente de aire necesaria en el túnel de viento, se requerirá conocer, una vez determinadas las dimensiones de este, las diversas pérdidas de presión producidas por cada uno de ellos, las cuales se deben a la viscosidad de fluido y son calculadas por la ecuación de Darcy-Colebrook [26]:

$$
\Delta P_{f}=\zeta \cdot \frac{L}{D} \cdot \rho \cdot \frac{v^{2}}{2}
$$

$\Delta P_{f}$ se refiere a la pérdida por fricción; $\zeta$ es el factor de friccion; L es la longitud del ducto; $\mathrm{D}$ es el diámetro hidráulico; $\rho$ es la densidad del fluido y $v$ la velocidad promedio.

Por lo tanto, es necesario emplear la correlación de Colebrook [27], para determinar el factor de fricción:

$$
\frac{1}{\sqrt{\zeta}}=2 \cdot \log _{10}\left(R_{e} \sqrt{\zeta}\right)-0.8
$$

La potencia del ventilador y su consumo eléctrico están ligados a las pérdidas de presión, la forma de la sección transversal del túnel de viento, la geometría de las partes que conforman el túnel de viento, al igual que sus longitudes; así se verán reflejadas en el cálculo de pérdidas. 
Otro tipo de pérdidas que deben ser estimadas, son dinámicas que resultan de las perturbaciones del flujo causados por equipos montados en los conductos, accesorios, cambios de dirección del flujo de aire y de área.

Para ellos es necesario determinar coeficiente de pérdida local. El coeficiente adimensional $\mathrm{K}$ es usado para definir la pérdida de presión en conductos, este coeficiente que tiene el mismo valor en corrientes dinámicas similares.

$$
K=\zeta \cdot \frac{L}{D}
$$

Donde, $L$ es la longitud del conducto y $\mathrm{D}$ es el diámetro hidráulico del conducto.

Para ello se determinan las pérdidas para cada uno de los componentes que lo conforman:

Se utilizó el Honeycomb el cual tiene como objetivo proveer una corriente constante y uniforme en toda la sección de prueba del túnel de viento y reducir la turbulencia. Para determinar las Pérdidas dinámicas, y determinar las pérdidas en el Honeycomb, la ecuación es la siguiente [13]:

$$
K=\lambda \cdot\left(\frac{L}{D_{h c}}+3\right)\left(\frac{A}{A_{\text {flow }}}\right)^{2}+\left(\frac{A}{A_{\text {flow }}}-1\right)^{2}
$$

Donde $\lambda$ es el coeficiente de fricción basado en el Honeycomb, $L$ es la longitud del conducto y $D_{\mathrm{hc}}$ es el diámetro hidráulico del conducto, $A$ hace referencia a la sección transversal, $A_{\text {flow }}$ se refiera el área de la sección transversal de flujo, y la estimación de $\lambda$ se realizó a través de la siguiente expresión:

$$
\lambda_{h}=\left\{\begin{array}{c}
0.375 \cdot\left(\frac{\Delta}{D}\right)^{0.4} \cdot \operatorname{Re}_{\Delta}^{-0.1} \text { para } e_{\Delta} \leq 275 \\
0.214 \cdot\left(\frac{\Delta}{D}\right)^{0.4} \text { para } \operatorname{Re}_{\Delta}>275
\end{array}\right.
$$

Donde $\Delta$ es la rugosidad de la superficie del Honeycomb, $\mathrm{R}_{\mathrm{e} \Delta}$ se refiere al número de Reynolds basado en la rugosidad del material y $D$ es el diámetro de la celda de Honeycomb.

También se requiere de la instalación de una malla posterior al Honeycomb. Su función principal es disipar la turbulencia de escala que el Honeycomb no puede disipar. El Honeycomb reduce o elimina los componentes de velocidad transversal, así como la turbulencia transversal. La misma malla es empleada en la parte delantera del ventilador, previendo protección instalada al mismo, la cual está situada antes del ventilador. Al estar produciendo pérdidas debido a la alta velocidad del flujo de aire en esta zona, aparte, provee control de separación de flujo.

Para determinar las pérdidas dinámicas a través de la malla que se encuentra en la parte posterior del Honeycomb; así como también la que se encuentra antes del ventilador, es posible emplear la siguiente expresión [4]:

$$
\begin{aligned}
& K_{m}=K_{\text {malla }} \cdot K_{R n} \cdot \sigma_{s}+\frac{\sigma_{s}^{2}}{\beta_{s}^{2}} \\
& K_{R n}=0.785 \cdot\left(\frac{R_{e w}}{241}+1.0\right)^{-4}+1.01 \\
& \beta_{s}=\left(1-d_{w} \cdot \rho_{m}\right)^{2} \\
& \rho_{m}=\frac{1}{W_{m}}
\end{aligned}
$$

Donde $\mathrm{K}_{\mathrm{m}}$ es la pérdida debido a la malla, $\mathrm{K}_{\text {malla }}$ es llamado factor, de malla, $K_{\mathrm{Rn}}$ es el factor de sensibilidad, $\sigma_{\mathrm{s}}$ es la solidés de la malla y $\beta_{\mathrm{s}}$ es la porosidad, $\rho_{\mathrm{m}}$ se refiere a la densidad de la malla, $\mathrm{W}_{\mathrm{m}}$ es el ancho de una celda de malla cuadrada y $\mathrm{d}_{\mathrm{W}}$ es el diámetro del alambre.

La pérdida debido a la fricción en la contracción puede ser estimada a través de la siguiente ecuación:

$$
\mathrm{K}_{\mathrm{ct}}=0.32 \cdot \zeta \cdot \frac{\mathrm{L}}{\mathrm{D}_{\mathrm{ct}}}
$$

Donde $\zeta$ es el factor de fricción, L es la longitud de la contracción y $\mathrm{D}_{\mathrm{ct}}$ es el diámetro hidráulico de la contracción y 0.32 es una constante de la ecuación.

Por otra parte, la pérdida debido a la contracción del túnel de viento debe cuantificarse. Por lo tanto, es importante determinar dicha pérdida a través de la siguiente expresión:

$$
K_{c t}=0.32 \cdot \zeta_{m} \cdot \frac{L_{d}}{D_{h c}}
$$

Donde $\zeta_{m}$ se refieren al factor de fricción, $\mathrm{L}_{\mathrm{d}}$ es la longitud de la contracción y $\mathrm{D}_{\mathrm{hc}}$ es el diámetro hidráulico de la sección de trabajo. El factor de fricción no es más que: 


$$
\zeta_{m}=\frac{\zeta_{f}+\zeta_{c t}}{2}
$$

Por lo tanto, es necesario emplear la correlación de Colebrook nuevamente.

La estimación de la pérdida de carga en las secciones constantes como la sección de prueba y la sección antes de ella se empleó la siguiente ecuación:

$$
K_{c t}=\zeta \cdot \frac{L_{d}}{D_{h c}}
$$

Donde $\zeta, \mathrm{L}_{\mathrm{d}}, \mathrm{D}_{\mathrm{hc}}$ se refieren al factor de fricción, la longitud de la contracción y el diámetro hidráulico de la sección de pruebas, respectivamente.

En el difusor, al igual que otros componentes de túnel de viento, produce pérdidas. La mecánica de fluidos de los difusores es compleja, ya que el flujo puede depender de los detalles de los perfiles de flujo entrantes, que pueden variar con las configuraciones específicas del modelo de nuestro diseño del túnel de viento; pero muchos autores [4][37], sugieren emplear las siguientes ecuaciones para determinar las pérdidas.

$$
\begin{gathered}
K_{D}=K_{f}+K_{\text {Exp }} \\
K_{f}=\left(1-\frac{1}{A_{R}^{2}}\right) \cdot \frac{\zeta}{8 \cdot \sin \theta} \\
K_{\text {Exp }}=K_{e}(\theta)\left(\frac{A_{R}-1}{A_{R}}\right)^{2} \\
A_{R}=\frac{A_{2}}{A_{1}}
\end{gathered}
$$

$\mathrm{K}_{\mathrm{e}}($ Cuadrada $)=0.1709-0.170 \cdot \theta+0.03260 \cdot$ $\theta^{2}+0.001078 \cdot \theta^{3}-0.0009076 \cdot \theta^{4}-0.00001331$. $\theta^{5}+0.00001345 \cdot \theta^{5}$

Donde $\mathrm{K}_{\mathrm{D}}$ se refiere a la pérdida del difusor, $\zeta$ es el factor de fricción, $\mathrm{K}_{\mathrm{f}}$ se refiere a la pérdida por fricción, $\mathrm{K}_{\mathrm{Exp}}$ no es más que la pérdida por la expansión, $\mathrm{K}_{\mathrm{e}}(\theta)$ es un factor el cual depende de la sección transversal del difusor empleado para determinar el factor de pérdida por la expansión empleada en una correlación cuyos límites de validés se encuentran entre $1.5^{\circ}$ y $5^{\circ}, A_{R}$ se refiere la relación de área, siendo $\mathrm{A}_{1}$ el área de entrada al difusor, $A_{2}$ es el área de salida del difusor, $\theta$ no es más que el ángulo del difusor.
El enderezador de flujo es empleado para eliminar la rotación del fluido. Lo que se persigue al emplearlo es recuperación de presión; para determinar la pérdida de presión del enderezador de flujo se emplea la siguiente ecuación:

$$
\begin{aligned}
& K_{t h}=\frac{2}{\lambda}\left(\varepsilon_{s}-\varepsilon_{p}\right) \\
& \lambda=\frac{u}{\Omega \cdot r} \\
& \varepsilon_{s}=\frac{w_{s} \cdot r}{u} \\
& \varepsilon_{p}=\frac{w_{p} \cdot r}{u}
\end{aligned}
$$

Donde $\varepsilon_{\mathrm{s}}$ es el coeficiente de torbellino a la salida del ventilador, $\varepsilon_{\mathrm{p}}$ es el coeficiente de torbellino a la entrada del ventilador y $\lambda$ no es más que el coeficiente de flujo, $u$ se refiere a la velocidad axial, $w_{\mathrm{p}} \mathrm{r}$, $\mathrm{w}_{\mathrm{s}} \mathrm{r}$ son los componentes tangenciales de la velocidad.

\subsection{Modelado Numérico CFD}

Esta sección presenta la descripción general del diseño de túnel de viento de circuito abierto y el modelado dinámico de fluidos computacional (CFD), empleando Ansys Fluent. El dominio computacional consta de cuatro dominios básicos que son: la contracción, la sección de prueba, el difusor y el ventilador. Como muestra, a continuación:

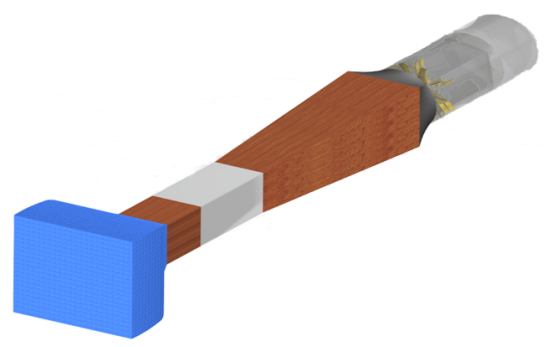

Figura 6. Dominio computacional.

El modelo CFD permitirá la obtención de información no suministrada por el diseñador y vendedor del ventilador, además de información muy importante como son los perfiles de velocidad en la zona de pruebas, los datos de velocidad axial antes y después del ventilador, que permita calcular los coeficientes de torbellinos; facilitando la evaluación de posibles zonas donde es posible la recirculación del aire, permitiendo evaluar y modificar la geometría inicial del diseño. 
Aparte de los cuatro dominios básicos, se incluyó en la simulación (CFD) la sección del ventilador, aumentando el tiempo de simulación y elementos empleados durante la misma.

Para ello se ha requerido realizar la discretización del dominio de cálculo el cual está conformado por los cuatro dominios básicos y la zona del ventilador, empleando para ellos volúmenes finitos y una malla ortogonal estructurada hexaédrica.

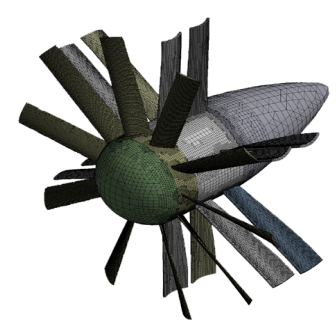

Figura 7. Discretización del ventilador axial.

Inicialmente se creó una malla estructurada hexaédrica ortogonal con 900,000 celdas, posteriormente se procedió a realizar una independencia de malla [41] hasta obtener una malla final con 3,200,000 de celdas y un error de continuidad del fluido menor a $0.01 \%$.

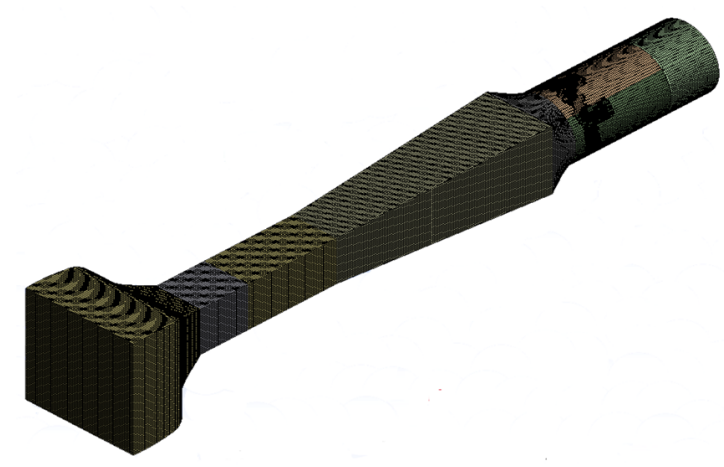

Figura 8. Discretización del dominio computacional.

Se emplearon inicialmente los modelos K-E (estándar) y K-w (sst); durante el proceso de independencia de malla, el modelo $\mathrm{K}-\mathcal{E}$ resultó más atractivo debido al tiempo de convergencia relativamente menor que el modelo K-w.

El modelo K- $\varepsilon$ [42] es un modelo ampliamente utilizado en CFD, emplea dos ecuaciones para describir turbulencia. Estas se emplean para definir la escala de velocidad $v$ y 1 para definir la longitud de la escala.

$$
\begin{aligned}
& v=K^{\frac{1}{2}} \\
& l=\frac{k^{\frac{3}{2}}}{\varepsilon}
\end{aligned}
$$

El modelo K- $\varepsilon$ estándar emplea cinco constantes de ajuste ya predefinidas las cuales son:

$\mathrm{C}_{\mathrm{u}}=0.09 ; \quad \sigma_{\mathrm{k}}=1.00 ; \sigma_{\varepsilon}=1.30 ; \mathrm{C}_{1 \varepsilon}=1.44 ; \mathrm{C}_{2 \varepsilon}=1.92$

Las anteriores constantes de ajuste de dicho modelo fueron obtenidas a través de experimentación y ajuste durante el desarrollo de este modelo [43]. Cabe resaltar que estos tipos de modelos están apoyadas en muchas suposiciones y simplificaciones; pertenecen a grupo de modelos clásicos empleados en CFD basados en el enfoque de soluciones de las ecuaciones de NavierStokes, promediadas de dichas ecuaciones.

El $\mathbf{y}+$ de esta malla es de 350 [41], ubicándonos en la zona donde la turbulencia predomina. Por ende no se está tratando de solucionar la capa límite en nuestro diseño preliminar; si no que será tema de los siguientes papers sobre este diseño preliminar. Resolver la capa límite o tener una aproximación requiere usar funciones de pared, requerirá de alto costo computacional por el refinamiento de la malla en la cercanía de las paredes a lo largo del túnel de viento.

\section{Resultado}

Los resultados siguientes no permiten conocer las pérdidas para cada sección del túnel de viento con el fin de seleccionar correctamente el ventilador axial que cubra todas las pérdidas del túnel de viento.

Tabla 3. Pérdida de presión por componentes del túnel de viento

\begin{tabular}{cccc}
\hline Componentes & Hl & HI (CFD) & $\%$ \\
& (Teórico) & & Error
\end{tabular}

$\begin{array}{llcc}\text { Contracción } & 0.355 & 0.3539 & 0.31 \\ \text { Adicional } & 44,24 & 40.72 & 7.8\end{array}$




\begin{tabular}{llll}
$\begin{array}{c}\text { Sección de } \\
\text { pruebas }\end{array}$ & 85.40 & 83.99 & 1.65 \\
& & & \\
Difusor & 43.68 & 49.12 & 11.08 \\
\hline
\end{tabular}

La anterior tabla muestra un error de los cálculos teóricos vs los experimentales. Los mayores errores se encuentran en el difusor debido a las turbulencias que se generan debido al ventilador. Por lo general, es el componente de mayor contribución de pérdidas en el túnel de viento. Se debe buscar su correcto dimensionamiento para mantener un flujo lo más uniforme posible en todas las áreas del túnel de viento y evitar separación de flujo.

En trabajos futuros debe mejorarse la malla del difusor, incluirse la cámara de tranquilización (honeycomb y sus mallas, las paredes del recinto donde se instalará el túnel de viento debe ser incluidas en la simulación). Esto permitirá un estudio más detallado del túnel de viento, así como optimizar la geometría, eliminar oscilaciones, flujo que podría llegar a ocurrir a medida que detalle el túnel de viento.

Se debe tener en cuenta la existencia de una gran cantidad de incertidumbre por parte de los fabricantes en cuanto a la rugosidad que presentan los materiales empleados en la simulación. Esto podría disminuir el error mostrado en la tabla 3.

También disminuir el $\mathrm{Y}+$ empleado en nuestro modelo $\mathrm{K}-\varepsilon$ hasta un rango $5<\mathrm{Y}+<40$, actualmente no encontramos con un $\mathrm{Y}+$ de 350 . Debemos aclarar que los resultados expuestos a la fecha en este rango expresan los componentes de velocidad de una manera eficaz; resultado de correlacionar el perfil de velocidad basada en la teoría existente a la fecha [44] [45].

El resultado de la simulación en la imagen nos muestra una relación de presión constante en la mayor parte del túnel de viento. Los reportes sobre caída de presión en el túnel de viento muestran un promedio de 2335.7889 $\mathrm{Pa}$ que equivale a $0.337 \mathrm{psi}$, una diferencia con nuestros datos teóricos de 0.38 psi, en condiciones de velocidad en la sección de prueba de $65 \mathrm{~m} / \mathrm{s}$.

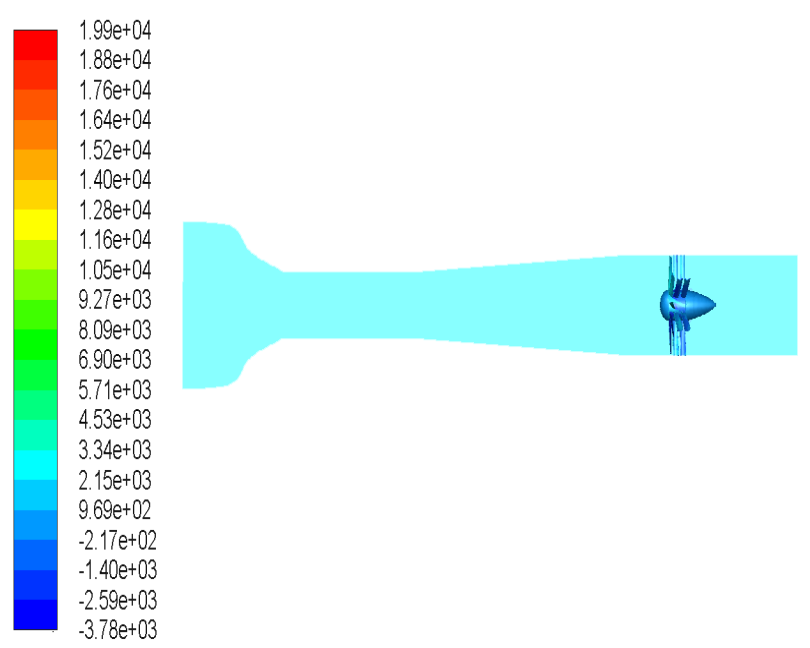

Figura 9. Relación de Presión en el Túnel de Viento.

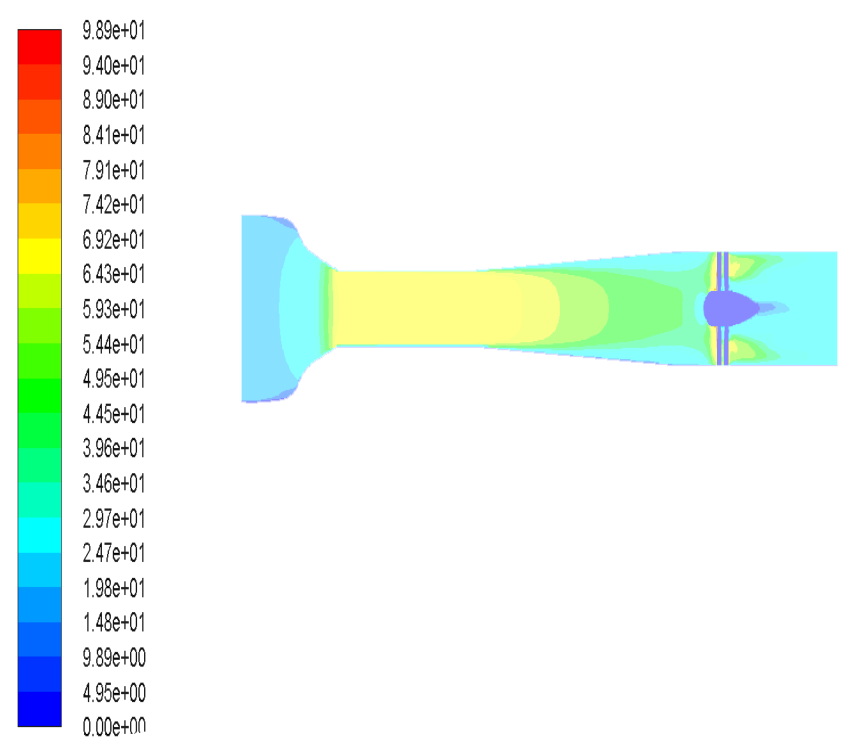

Figura 10. Velocidades en el interior del túnel de viento.

En la imagen observamos el desarrollo del perfil de velocidades para la condición de velocidad $65 \mathrm{~m} / \mathrm{s}$. Se observa claramente un perfil de velocidad desarrollado para esta condición de interés. Dicho perfil está desarrollado desde la contracción, sin ningún tipo de perturbación debido al correcto dimensionamiento, no solo de la contracción sino del conjunto completo. 


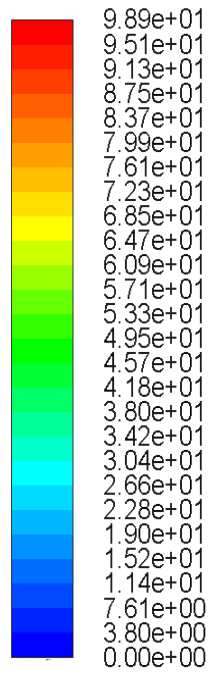

Figura 11. Velocidad a la entrada a la sección de pruebas.
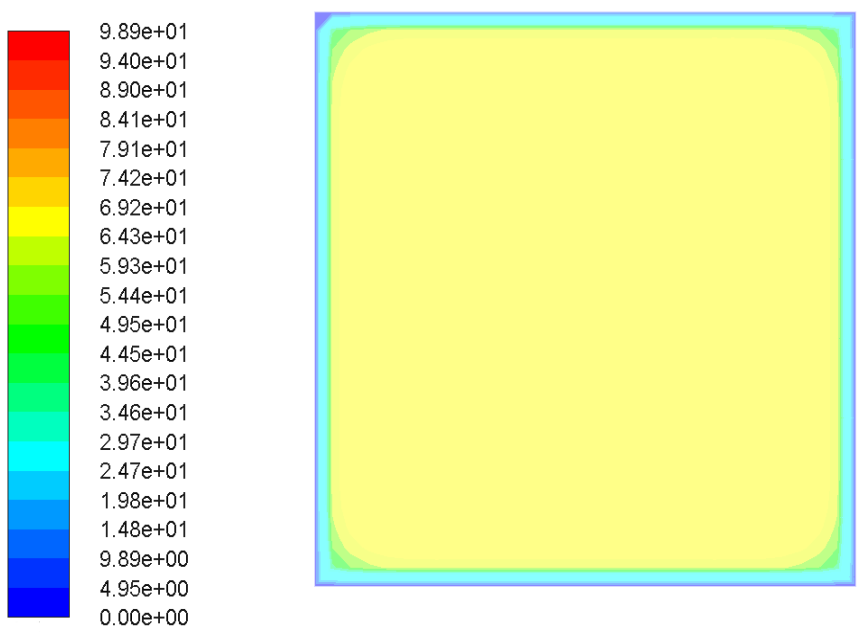

Figura 12. Velocidad a la salida a la sección de pruebas.

Se observa una disminución de la velocidad entre salida $(62.9 \mathrm{~m} / \mathrm{s})$ y la entrada $(68.5 \mathrm{~m} / \mathrm{s})$ de la sección de pruebas (figuras 11 y 12). El patrón visual de la distribución de velocidad en ambas secciones muestra claramente uniforme $\mathrm{y}$, a medida que el fluido se acerca a la pared, una disminución de dicha velocidad. Las esquinas de dicha sección poseen velocidades muy cercanas a cero.

En la imagen anterior se observa el punto de operación del ventilador (punto azul). La curva en negro hace referencia al sistema mientras que la curva en rojo es el caudal del ventilador vs su presión diferencial. El ventilador cumple con las pérdidas en el túnel de viento.

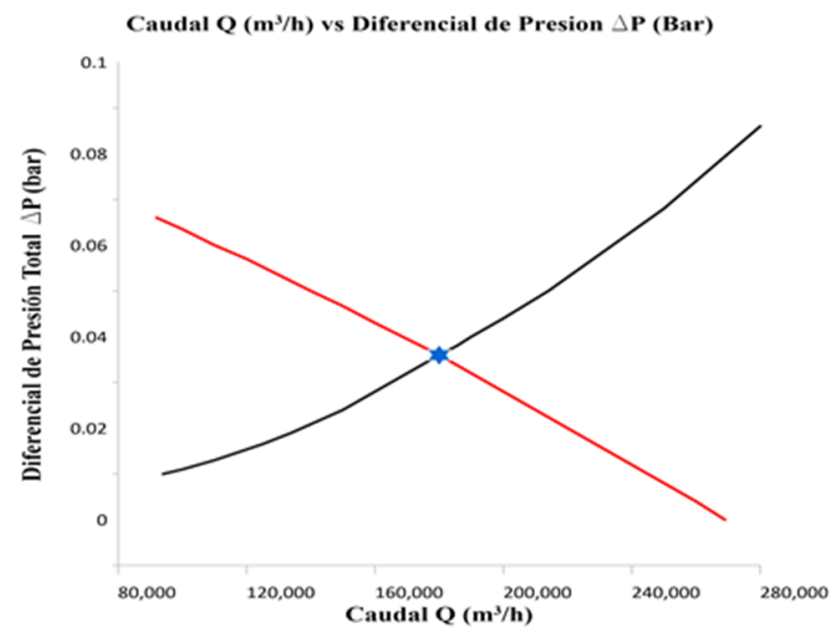

Figura 13. Curva Característica del ventilador axial caudal (m3/s) vs diferencia de presión (bar).

En la imagen anterior se observa el punto de operación del ventilador (punto azul). La curva en negro hace referencia al sistema mientras que la curva en rojo es el caudal del ventilador vs su presión diferencial. El ventilador cumple con las pérdidas en el túnel de viento.

\section{Conclusiones}

- Se desarrolló una nueva correlación para el diseño de contracción para túnel de viento abierto, ecuación (1).

- El empleo de la dinámica de fluido computacional (CFD) permitió evaluar dicha correlación y obtener datos no facilitados por el fabricante del ventilador axial.

- La dinámica de fluido computacional (CFD) para determinar la presión necesaria que debe proveer el ventilador axial para solventar todas las pérdidas en nuestro túnel de viento, muestra claramente que es $11.31 \%$ menor que las estimadas teóricamente, ya que no toma en cuenta las pérdidas por fugas que puedan darse.

- La poca documentación sobre el diseño básico de ventiladores axiales empleados para este fin.

- La baja eficiencia del ventilador $68.3 \%$ hace necesario un diseño futuro.

- Las siguientes partes de esta publicación permitirán incorporar el análisis de capa límite no desarrollado en este manuscrito (papers), al igual que mostrar perfiles de velocidad más detallados e incluir gráficas de velocidad a lo largo de la sección de prueba y túnel de viento, tener en cuenta aspectos como Honeycomb, malla y las dimensiones posibles de sitio donde se 
llevará a cabo su construcción con el fin de poder simular las paredes, toma de aire, etc.

- El diseño final y su construcción permitirá la realización de diversas investigaciones relacionadas con mecánica de fluidos, aerodinámica, dispersión de emisiones contaminantes, cargas sobre edificaciones, etc.

\section{Agradecimiento}

Agradecemos al Doctor Oscar Garibaldi por sus acertadas sugerencias y de igual modo al Doctor John Sullivan. El reconocimiento a la Secretaría Nacional de Ciencia, Tecnología e Innovación (SENACYT) por el apoyo financiero.

\section{Referencias}

[1] C. A., Quispe, W. J., Urcuhuaranga, J. E. Chiroque. "Diseño Aerodinámico de un Túnel de Viento de Bajas Velocidades". Revista de Investigación de Física vol. 17, 1417001601, 2014.

[2] G. Johl, M. Passmore, P. Render. "Design Methodology and Performance of an Indraft Wind Tunnel". The Aeronautical Journal, vol. 108, pp. 465-473, 2004.

[3] R.C. Pankhurst, D.W. Holder. "Wind Tunnel Technique an Account of Experimental Methods in Low and High Speed Wind Tunnels". Pitman \& Sons, 1952, pp. 117-130.

[4] J.B. Barlow, W.H. Rae, A. Pope. "Low Speed Wind Tunnel Testing”. John Wiley \& Sons, 1999, pp. 19-120.

[5] P. Bradshaw, R.C. Pankhurst. "The Design of Low Speed Wind Tunnel". Progress in Aerospace Sciences, vol.5, pp 1-69, 1964.

[6] B. Celis, H. H. Ubbens. "Design and Construction of an Open Circuit Wind Tunnel with Specific Measurement for Cycling”. Procedia Engineering, vol. 147, pp. 98-103, 2016.

[7] N.J. cook. "A Boundary Layer Wind Tunnel for Building Aerodynamics". Journal Industrial Aerodynamics, vol. 1, pp. 312, 1975.

[8] J.E. Cermak. "Wind Tunnel development and Trends in Applications to Civil Engineering". Journal of Wind Engineering and Industrial Aerodynamics, vol. 91, pp. 355-370, 2003.

[9] H. A. Al Nehari, A.K. Abdel Rahman, A. El Moneim Nassib, H.M. Shafey. "Design and Construction of a Wind Tunnel for Enviromental Flow Studies". Journal of Engineering Sciencies, Assiut University, vol. 38, pp. 177-193, 2010.

[10] G. Dianna, S. De Ponteb, M. Falcoa, A. Zassoa. “ A New Large Wnd Tunnel for Civil Environmental and Aeronautical Applications". Journal of Wind Engineering and Industrial Aerodynamics, vol. 74, pp. 553-565, 1998.

[11] M. A. Gonzalez Hernandez, A.I. Moreno López, A., Artur, J.M. Perales Perales, Y. Wu, S. Xiaoxiao. "Design Methodology for a
Quick and Low-Cost Wind Tunnel". Wind Tunnel and Their Diverse Engineering Applications, Intech, 2013.

[12] B.H. Goethert. "Transonic Wind Tunnel Testing". Dover Publications, 2007, pp. 2-29.

[13] W. T. Eckert, K.W. Mort, J. Jope. "Aerodynamic Design Guidelines and Computer Program for Estimation of Subsonic Wind Tunnel Performance". Nasa TN D-8243, 1976.

[14] R.D. Metha, P. Bradshaw. "Design rules for Small Low Speed Wind Tunnels". The Aeronautical Journal, vol.83, pp. 443-449, 1979.

[15] J.H. Bell, R.D. Metha. "Boundary Layer Predictions for Small Low Speed Contractions”. AIAA, vol. 27, pp. 372-374, 1989.

[16] D.Brassard, M. Ferchichi. Transformation of a Polynomial for a Contraction Wall Profile". ASME Journal Fluid Engineering, vol. 127 (1), pp. 183-185, 2005.

[17] J. H. Watmuff. "Wind Tunnel Contraction Design". 9th Australasian Fluid Mechanics Conference, Auckland, pp. 8-12, 1986.

[18] R. Harrop. "A Method for Designing Wind Tunnel Contractions". Journal of the Aeronautical Society, vol.55, pp 169-180, 2016.

[19] A.S Abdelhamed, Y. El-S Yassen, M.M. ElSakka. "Design Optimization of Three Dimensional Geometry of Wind Tunnel Contraction". Ain Shams Engineering Journal, vol. 6, pp. 281$288,2015$.

[20] L. Leafsome, S. Koziel, F. Andrason, K. Magnusson, A. Gylfason. "Numercial Optimization and Experimental Validation of a Low Speed Wind Tunnel Contraction”. Procedia Computer Science vol. 9, pp.822-831, 2012.

[21] N. Nordin, Z.A. Abdul Karim, S. Othman and V. R. Raghavan. "Design and Development of Low Subsonic Wind Tunnel for Turning Diffuser Application. Advanced Materials Research, vol. 614-615, pp. 586-591, 2012.

[22] Q.Y. Nguyen. "Designing, Construction and Testing a Low Speed Open Jet Wind Tunnel". Journal of Engineering Research and Applications, vol. 4, pp. 243-246, 2014.

[23] Y.H. Kao, Z.W. Jiang, S.C. Fang. "A computational Simulation Study of Fluid Mechanics of Low Speed Wind Tunnel Contractions". Fluid, vol. 2, 23, 2017.

[24] A. Luszczewski. "Redes industriales de tubería, bombas para agua, ventiladores y compresores”. Reverte, 1999, pp. 223-277.

[25] V. Dakshina Murty. “Turbomachinery Concepts, Applications and Design". CRC press, 2018.

[26] C.F. Colebrook. Turbulent Flow in Pipes, with Particular Reference to the Transition between the Smooth and Rough Pipe Laws". Journal of the Institute of Civil Engineers, vol.11, pp. 133-156, 1939.

[27] C.F. Colebrook, M. White. "Experiments with Fluid in Roughened Pipes". Proceeding of the Royal of London. Series 
A, Mathematical and Physical Sciences, vol. 161 (906), pp. 367381, 1937.

[28] A. Betz. "Introduction to the Theory of Flow Machines". Pergamon Press, 1966, pp. 161-167.

[29] J.C. Páscoa. "Turbomaquinas - Uma Abordagem Moderna". Engebook, 2017, pp. 147-181.

[30] R.A. Wallis. “Axial Flow Fans Design and Practice”. Academic Press, 1961.

[31] S.L. Dixon \& C., Hall. "Fluid Mechanics and Thermodynamics of Turbomachinery". Butterworth-Heinemann, 2014, pp. 265316.

[32] E. Logan. "Turbomachinery: Basic Theory and Applications". CRC Press, 1993, pp. 109-136.

[33] A.J. Stepanoff. "Turboblowers: Theory, Design and Application of Centrifugal and Axial Flow Compressor and Fans". John Wiley \& Sons, 1955, pp. 277-308.

[34] E. Dick. "Fundamental of Turbomachines". Springer, 2014, pp. 97-151.

[35] T. Wright and P.M., Gerhart. "Fluid Machinery". CRC Press, 2009, pp. 125-149.

[36] S.M. Yahya. "Turbines, Compressors and Fans". Tata Mc Graw Hill, 2011, pp. 639-666.

[37] G. A. pignone, U. R. Vercelli. "Turbomacchine. Elementi Teorici e Progettuali-Compressori-Turbine-Ventilatori-Eliche". Ulrico Hoepli, 1991, pp. 123-207.

[38] M.P. Encinas. "Turbomaquinas Hidraulicas". Limusa, 1976, pp.176-193.

[39] M.P. Encinas. "Turbomaquinas de Fluido Compresible: Turbocompresores, Turbinas de Gas, Turbinas de Vapor". Limusa, 1984, pp. 125-198.

[40] R.D. Mehta. The Aerodynamic Design of Blower Tunnels with Wide Angle Diffusers". Progress Aerospace Science, vol. 18, pp. 59-120, 1979.

[41] H.K. Versteeg \& W. Malalasekera. "An Introduction to Computational Fluid”. Prentice Hall, 1995, pp. 72-90.

[42] J.M. Fernández Oro. "Técnicas numéricas en ingeniería de fluidos". Reverte, 2007, pp. 284-307.

[43] B.E. Launder, D.B. Spalding. "The Numerical Computational of Turbulent Flows". Computer Methods in Applied Mechanics and Engineering, vol. 3, pp.269-289, 1974.

[44] P.A. Durbin , B.A. Pettersson Reif. " Statistical Theory and Modelling for Turbulent Flows. John Wiley \& sons, 2003, pp. 59-70.

[45] P.A. Davidson. "Turbulence: An Introduction for Scientists and Engineers”. Oxford University Press, 2007, pp. 107-131.

\section{Siglas}

$\boldsymbol{\mu}=$ Viscosidad dinámica el aire

$\boldsymbol{\rho}=$ Densidad del aire

$\mathbf{v}=$ Velocidad del aire

D = Diámetro hidráulico

$\mathbf{R e}=$ Número de reynolds

$\boldsymbol{\Theta}_{\mathbf{e}}=$ Angulo del difusor

$\mathbf{A}_{\mathbf{R}}=$ Relación de áreas para el difusor

$\boldsymbol{\eta}=$ Eficiencia del ventilador axial

$\boldsymbol{\varepsilon}_{\mathbf{m}}=$ Indicie de frotamiento

$\boldsymbol{\varphi}=$ Coeficiente de flujo del ventilador axial

$\psi=$ Coeficiente de trabajo del ventilador axial

$\eta_{\mathbf{p}}=$ Eficiencia isentropica

$\boldsymbol{\eta}_{\text {vol. }}=$ Eficiencia volumétrica del ventilador axial

$\boldsymbol{\eta}_{\text {mec. }}=$ Eficiencia mecánica del ventilador axial

$\boldsymbol{\eta}_{\text {aero. }}=$ Eficiencia aerodinámica del ventilador axial

$\Delta \mathbf{P}=$ Diferencia de presión

$\Delta \mathbf{P}_{\zeta}=$ Pérdida debido a la fricción

$\mathbf{K}=$ Coeficiente adimensional de Pérdida local

$\mathbf{L}=$ longitud del conducto

$\lambda=$ Coeficiente de fricción del Honeycomb / coeficiente de flujo

$\mathbf{A}_{\text {flow }}=$ Área de sección transversal de flujo

$\boldsymbol{\Delta}=$ Rugosidad de la superficie del Honeycomb

$\mathbf{R}_{\mathbf{e} \Delta}=$ Numero del Reynolds basado en la rugosidad del material

$\mathbf{D}_{\mathbf{h c}}=$ Diámetro Hidráulico del Honeycomb

$\mathbf{K}_{\mathbf{m}}=$ Es la Pérdida debido a la malla 
$\mathbf{K}_{\text {malla }}=$ Factor de malla

$\mathbf{K}_{\mathbf{R n}}=$ Factor de sensibilidad

$\boldsymbol{\sigma}_{\mathrm{s}}=$ Solides de la malla

$\boldsymbol{\beta}_{\mathbf{s}}=$ Porosidad

$\rho_{\mathbf{m}}=$ Densidad de la malla

$\mathbf{W}_{\mathbf{m}}=$ Ancho de una celda de malla cuadrada

$\mathbf{d}_{\mathbf{w}}=$ Diámetro del alambre

$\mathbf{D}_{\mathrm{ct}}=$ Diámetro hidráulico de la contracción

$\zeta_{\mathrm{m}}=$ Factor medio de fricción

$\mathbf{K}_{\mathbf{D}}=$ Pérdida del difusor
$\mathbf{K}_{\mathbf{f}}=$ Pérdida por fricción

$\mathbf{K}_{\mathbf{E x p}}=$ Pérdida por expansión

$\mathbf{K}_{\mathbf{e}}(\boldsymbol{\theta})=$ Factor de perdida dependiente de la sección transversal del difusor

$\varepsilon_{\mathrm{s}}=$ Coeficiente de torbellino

$\boldsymbol{\varepsilon}_{\mathbf{p}}=$ Coeficiente de torbellino a la entrada del ventilador

$\mathbf{u}=$ Velocidad axial

$\mathbf{w}_{\mathbf{p}} \mathbf{r}=$ Componente tangencial de velocidad antes del rotor $\mathrm{W}_{\mathrm{s}} \mathrm{r}=$ Componentes tangencial de velocidad justo delante del rotor. 